\section{International Scientific Journal Theoretical \& Applied Science}

p-ISSN: 2308-4944 (print) e-ISSN: 2409-0085 (online)

Year: 2018 Issue: 09 Volume: 65

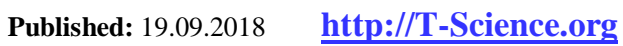

SECTION 2. Applied mathematics.

Mathematical modeling.
Zafar Ravshanov

Researcher,

Tashkent University of Information Technologies,

Tashkent, Uzbekistan

Nodira Tashtemirova

Researcher,

Tashkent University of Information Technologies,

Tashkent, Uzbekistan

Abduvali Mukhamadiev

Head of Department,

Tashkent University of information Technologies,

Tashkent, Uzbekistan

\title{
ADVANCED MATHEMATICAL MODEL OF THE PROCESS OF FINE- DISPERSED PARTICLES DISTRIBUTION IN THE ATMOSPHERE
}

Abstract: The paper considers the urgent task related to monitoring and forecasting the ecological state of the surface layer of the atmosphere in industrial regions with the aim of maintaining a balance of the sanitary norm. The review of scientific works on the development of mathematical models, numerical methods and their software for carrying out a complex study of transport processes, diffusion and transport of aerosol particles and exhaust gases in the atmosphere is reviewed. The developed mathematical model of the process of transport and diffusion of aerosol particles in the atmosphere is considered, which takes into account the essential parameters of the research object: the velocity of the particles of aerosol emissions in the atmosphere, which depends on the mass and radius of the particles; acceleration of free falling of particles; coefficient of the drag of particles during their transfer by air mass; body shape factor for calculating the drag force; air flow lift; the density of particles and air, as well as the viscosity of air.

Key words: mathematical model, transport, diffusion, harmful substances, weather and climate factors, hydromechanics, fine particles.

Language: Russian

Citation: Ravshanov Z, Tashtemirova N, Mukhamadiev A (2018) ADVANCED MATHEMATICAL MODEL OF THE PROCESS OF FINE-DISPERSED PARTICLES DISTRIBUTION IN THE ATMOSPHERE. ISJ Theoretical \& Applied Science, 09 (65): 63-69.

Soi: http://s-o-i.org/1.1/TAS-09-65-11 Doi: crostef https://dx.doi.org/10.15863/TAS.2018.09.65.11

\section{УСОВЕРШЕСТВОВАННАЯ МАТЕМАТИЧЕСКАЯ МОДЕЛЬ ПРОЦЕССА РАСПРОСТРАНЕНИЯ МЕЛКОДИСПЕРСНЫХ ЧАСТИЦ В АТМОСФЕРЕ}

Аннотация: В работе рассматривается актуальная задача, связанная с мониторингом и прогнозированием экологического состояния приземного слоя атмосферы промышленных регионов с иелью сохранения баланса санитарной нормы. Приведен обзор научных работ по разработке математических моделей, численных методов и их программного обеспечения для проведения комплексного исследования прочессов переноса, диффузии и транспорта аэрозольных частиц и выбросных газов в атмосфере. Рассматривается разработанная математическая модель процесса переноса и диффузии аэрозольных частиц в атмосфере, в которой учтены существенные параметры объекта исследования: скорость перемещения частиц аэрозольных выбросов в атмосфере, зависящая от массы и радиуса частиц; ускорение свободного падения частиц; коэффициент лобового сопротивления частиц при переносе их воздушной массой; коэффициент формы тела для вычисления силь сопротивления; подъемная сила воздушного потока; плотность частии и воздуха, а также вязкость воздуха.

Ключевые слова: математическая модель, перенос, диффузия, вредные вещзества, погодноклиматические факторы, гидромеханика, мелкодисперсные частицы.

\section{Введение}

Вопросы мониторинга, прогнозирования и оценки загрязнения атмосферы и подстилающей поверхности земли пассивными и активными примесями, мелкодисперсными частицами и углекислыми газами, а также вопросы проектирования и размещения промышленных объектов с соблюдением предельно допустимых 
санитарных норм для промышленных регионов составляют собой одну из актуальных проблем в области охраны окружающей среды.

Анализ состояния окружающей среды за последние годы показал, что большой рост выброса вредных веществ в атмосфере привел к дисбалансу экологического состояния отдельных регионов. Это особенно заметно в государствах, где имеет место высокий темп развития промышленности, например, Китай, Россия, Индия, США, Франция, Великобритания, Япония, Северная Корея, Малайзия и др.

Негативные последствия возникают за счет увеличения загазованности атмосферы и концентрации вредных мелкодисперсных частиц, отрицательно воздействуя на живую систему флору и фауну регионов, а на глобальном уровне вносят лепту в изменение климата земного шара.

Надо отметить, что для комплексного исследования указанной проблемы необходимо создание конструктивной системной методологии математического моделирования, использование которой даст возможность проведения мониторинга и прогнозирования экологического состояния промышленных регионов с целью предотвращения опасных техногенных последствий и принятия правильных управленческих решений.

\section{Обзор литературы}

Научные исследования, направленные на разработку математических моделей и вычислительных алгоритмов для систем мониторинга и прогнозирования загрязнения атмосферы промышленных регионов, проводятся в передовых научных центрах и высших учебных заведениях мира, в число которых входят Karlsruhe Institute of Technology, Institute of Applied Geosciences (Германия), Centre for Ecology and Hydrology, Centre for Water Science (Великобритания), Finnish Meteorological Institute (Финляндия), National Institute of Hydrology, Waterloo Hydro Geologic (Индия), Институт атмосферы физики им. А.М. Обузова, Институт вычислительной математики и математической геофизики (Россия), Научно-исследовательский и проектно-технологический институт «Атмосфера», Национальный университет Узбекистана, Ташкентский университет информационных технологий (Узбекистан) и других.

В частности, проблемой математического моделирования процессов переноса, диффузии и транспорта вредных веществ (углекислые газы, мелкодисперсные аэрозольные пассивные и активные частиц) занимаются научные школы, созданные под руководством Г.И. Марчука, В.В. Пененко, А.Е. Алояна, Л.Т. Матвеева, В.П. Дымникова И.Э. Нааца, Э.А. Закарина, И.А.
Кибеля, Л.Н. Гутмана, Ф.Б. Абуталиева, а также зарубежных ученых W.J. Layton, J.H. Ferziger, J.W. Deardorff, M. Germano, U. Piomelli, L.C. Berselli, G.S. Winckelmans, W.C. Reynolds, X. Зидиск, К.А. Велдс, К.И. Наппо, Ж. Готаас, М. Мюллиоланд, С. Трап, М. Матиес, В. Эдельман и др.

Фундаментальные аспекты методологии математического моделирования процесса переноса и диффузии вредных мелкодисперсных частиц и углекислых газов в атмосфере сформулированы в работах М.Е. Берлянда, Е.Л. Гениховича, Р.И. Оникула, Н.Л. Бызовой, Ю.А. Анохиной, А.Х. Остромогильского, Т Iversen., T.E. Nordeng, R.Lange, M Pekar., академика C.A. Солодкова и др.

Разработкой автоматизированных систем экологического мониторинга и прогнозирования состояния окружающей среды занимаются проф. Г.В. Аверин, А.А. Любимов, В.Ю. Волков, Ю.Д. Эдельштейн, В.В. Бугровский, А.М. Погорелов, А.В. Бизикин и др.

В работах М.Л. Арушанова и его учеников проведены подробные исследования, связанные процессами выноса соле-пылевых частиц из осушенной части Аральского региона и показано, на сколько изменяется климат региона вследствие воздействия выше указанного фактора [1-4]. Воздействие арозолей на климатические характеристики классифицировано следующим образом:

- увеличение температуры поверхности поглощающими аэрозолями;

- снижение температуры поверхности рассеивающими аэрозолями;

- уменьшение температуры поверхности вследствие увеличения количества облаков и поглощения ими прямой радиации;

- увеличение количества и продолжительности (вследствие задержки выпадения осадков) облачного покрова при больших концентрациях водорастворимого аэрозоля;

- увеличение количества осадков при небольших, но достаточных для образования крупных капель, концентрациях грубодисперсного аэрозоля (гигантских ядер конденсации).

Диссертация Пушилина Ю.Н. [5] посвящена проблеме математического моделирования процессов загрязнения атмосферы и решению таких задач, как прогнозирование, размещение постов для мониторинга (стационарных и мобильных), оценка вклада конкретного промышленного объекта в общее загрязнение воздуха в реальном времени с целью принятия управленческих мер по нормированию выбросов.

В статье [6] разработана модель 3D CFD для исследования процесса распространения мелкодисперсных аэрозольных частиц в 
атмосфере. Математическая модель основана на модели транспортного градиента. В работе приводятся подробный анализ результатов проведенных численных расчетов на ЭВМ и выводы связанные с ним.

В диссертационной работе Серёгина О.В. [7] разработаны математические модели поглощения атмосферного кислорода, валовых выбросов углекислого газа и радона в приземный слой атмосферы, отличающиеся тем, что учтено влияние протяженных газоотдающих поверхностей, контактирующих с вентиляционными струями и аэрогазодинамических характеристик очистных и подготовительных участков. Усовершенствована методика инвентаризации источников пылегазовых выбросов от угольных шахт Подмосковного бассейна, а также разработан комплекс программных средств, позволяющий автоматизировать процесс расчета пылегазовых выбросов в приземные слои атмосферы от источников поверхностного технологического комплекса угольных шахт, проводить вычислительные эксперименты при моделировании различных геоэкологических и геотехнологических условий.

В статье [8] разработана динамическая многомерная математическая модель тепломассопереноса вредных примесей и теплоты в производственной среде с активной вентиляцией и источниками загрязнения, которая в отличие от существующих моделей распространения производственных вредностей позволяет учитывать завихрения газо-воздушных потоков.

В работе [9] описан новый алгоритм извлечения полного содержания атмосферных тонкодисперсных частиц (частиц размером менее 1,0 и 2,5 мкм) из мультиспектральных спутниковых изображений в видимой и ИКобластях электромагнитного спектра. Алгоритм основан на регрессионных соотношениях между отражательной способностью верхней поверхности атмосферы, микрофизическими параметрами аэрозоля и геометрическими параметрами спутниковой сцены. Предлагаемый алгоритм реализован и протестирован для спутникового прибора MERIS (среднее разрешение изображения).

В работе [10] предлагается двухмерная модель адсорбции следовых атмосферных составляющих аэрозольных частиц в воздухе, выброшенные промышленным источником. Модель основана на применении теории турбулентной диффузии в пограничном слое атмосферы в сочетании с моделью дисперсии вредных газов и моделью адсорбции газа пористыми твердыми частицами. Профили скорости ветра, используемые в симуляциях, были установлены по данным, ранее полученных в полевых условиях авторами статьи в Северном Негрееве (Израиль). Разработанная модель позволяет анализировать пространственную и временную эволюцию концентрации адсорбента в газовой фазе, а также в твердых частицах.

Распределение концентрации адсорбента рассчитывается для твердых частиц РМ2.5-10, что типично для промышленных выбросов. Анализ проводится для различных метеорологических условий и классов устойчивости атмосферы. Показано, что концентрация газов, адсорбированных аэрозольным плюмом, сильно зависит от уровня атмосферной турбулентности. Полученные результаты сравниваются с имеющимися экспериментальными данными.

В работе [11] использованы модель COSMOART для прогноза концентраций загрязняющих веществ в атмосфере. Как отмечают авторы статьи система КОСМО-Ru7-AРТ способна адекватно смоделировать значение концентрации примесей в атмосфере. Система включает в себя модуль оценки выбросов загрязняющих веществ в атмосферу из лесных пожаров, который был успешно протестирован на случае лесных пожаров, произошедших летом 2010 года для европейской части России. В статье показано, что точный прогноз концентрации загрязняющих веществ оказывает положительное влияние на прогноз температуры воздуха из-за учета аэрозольной обратной связи на излучение.

В работе [12] получено точное аналитическое решение уравнения адвекциидиффузии, предполагающее параметризацию турбулентности для гауссовской дисперсии загрязнения и учет очистки осадков аэрозолями дождем. Влияние распределения размеров капель дождя было учтено с помощью метода МонтеКарло. Как отмечают авторы работы, предложенный подход позволяет анализировать пространственную и временную эволюцию концентрации аэрозоля в газовой фазе, а также в дождевых каплях. В работе получены явное аналитическое выражение, которое позволяет анализировать зависимость скорости аэрозолей ниже облака, абсорбирующих от гауссовых плюсовых выбросов воздуха по разным параметрам и обнаружено, что максимальная концентрация аэрозолей на уровне земли зависит от интенсивности осадков, а местоположение максимума приближается к источнику излучения, когда интенсивность осадков увеличивается. Сравнение предсказаний теоретической модели с экспериментальными данными, показало довольно хорошее согласие между теоретическими результатами и экспериментами. Как отмечают авторы статьи, полученные результаты могут быть полезны при анализе 


\begin{tabular}{|c|c|c|c|c|c|c|}
\hline Impact Factor: & $\begin{array}{l}\text { ISRA (India) } \\
\text { ISI (Dubai, UAE } \\
\text { GIF (Australia) } \\
\text { JIF }\end{array}$ & $\begin{array}{l}=1.344 \\
=0.829 \\
=0.564 \\
=1.500\end{array}$ & $\begin{array}{l}\text { SIS (USA) } \\
\text { PИНЦ (Russia) } \\
\text { ESJI (KZ) } \\
\text { SJIF (Morocco) }\end{array}$ & $\begin{array}{l}=0.912 \\
=0.156 \\
=4.102 \\
=2.031\end{array}$ & $\begin{array}{l}\text { ICV (Poland } \\
\text { PIF (India) } \\
\text { IBI (India) }\end{array}$ & $\begin{array}{l}=6.630 \\
=1.940 \\
=4.260\end{array}$ \\
\hline
\end{tabular}

различных моделей метеорологии и химии, включая очистку аэрозолей в пламени загрязнения воздуха до дождя и оценку воздействия на человека различных химических, биологических и радиологических загрязнителей.

Проведенный анализ выше указанных работ показали, что при математическом моделировании процесса переноса и диффузии вредных веществ в атмосфере не рассмотрены с учетом следующих основных факторов, которые существенно воздействуют на объект исследования:

при процессе переноса и диффузии вредных веществ в атмосфере не рассмотрен транспорт аэрозольных частиц через границы области решения как третья краевая задача;

- при математическом моделировании процесса в всех работах коэффициент поглощения вредных веществ в атмосфере, который должен изменятся со временем, брался постоянным;

- $\quad$ не учитывалась орографическая поверхность земли, которая играет существенную роль в процессе распространения аэрозольных частиц в атмосфере;

- не учитывались погодно-климатические факторы в реальном масштабе времени;

- во всех разработанных математических моделях процесса скорость и направление ветра брались постоянными, а анализ метрологических данных показал, что в среднем за сутки направление ветра и скорость изменялись 12-13 раз;

- в математических моделях процесса при переносе частиц задавались скорость воздушного потока в атмосфере, а не скорости перемещения частиц, а коэффициент взаимодействия с подстилающей поверхностью задавался постоянным, в то время, когда он зависит от физико-механических свойств поверхности земли;

- в математических постановках задачи скорость осаждения частиц задавалась как постоянная константа, она не изменялась со временем и в зависимости от размеров и формы частиц.

\section{Постановка задачи}

Для разработки адекватной математической модели процесса переноса и диффузии аэрозольных выбросов в атмосфере, где учитывается скорость перемещения частиц под действием скорости ветра в пограничном слое атмосферы, рассмотрим полное уравнение гидродинамики

$$
\left.\begin{array}{c}
\frac{\partial \theta}{\partial t}+u \frac{\partial \theta}{\partial x}+v \frac{\partial \theta}{\partial y}+\left(w-w_{g}\right) \frac{\partial \theta}{\partial z}+\sigma \theta= \\
=\mu\left(\frac{\partial^{2} \theta}{\partial x^{2}}+\frac{\partial^{2} \theta}{\partial y^{2}}\right)+\frac{\partial}{\partial z}\left(k(z) \frac{\partial \theta}{\partial z}\right)+ \\
+\delta Q(x, y, z, t) ; \\
m \frac{d u}{d t}=c_{f} \pi r^{2} \rho_{\theta}(u-U)^{2} ; \\
m \frac{d v}{d t}=c_{f} \pi r^{2} \rho_{\theta}(v-U)^{2} ; \\
m \frac{d w_{g}}{d t}=-\frac{4}{3} \pi r^{3}\left(\rho_{n}-\rho_{\theta}\right) g- \\
\quad-k_{f} \mu_{\theta} \pi r w_{g}+F_{n}
\end{array}\right\}
$$

с соответствующими начальным

$$
\begin{gathered}
\theta(x, y, z, 0)=\theta_{0}(x, y, z) ; \\
u=u(0) ; v=v(0) ; \\
w_{g}=w_{g}(0) \text { при } \mathrm{t}=0
\end{gathered}
$$

и краевыми условиями

$$
\begin{aligned}
& -\left.\mu \frac{\partial \theta}{\partial x}\right|_{x=0}=\eta\left(\theta_{a}-\theta\right) ; \\
& \left.\mu \frac{\partial \theta}{\partial x}\right|_{x=L_{x}}=\eta\left(\theta_{a}-\theta\right) ; \\
& -\left.\mu \frac{\partial \theta}{\partial y}\right|_{y=0}=\eta\left(\theta_{a}-\theta\right) ; \\
& \left.\mu \frac{\partial \theta}{\partial y}\right|_{y=L_{y}}=\eta\left(\theta_{a}-\theta\right) ; \\
& -\left.\kappa(z) \frac{\partial \theta}{\partial z}\right|_{z=0}=\left(\beta \theta-Q_{0}\right) ; \\
& \left.\kappa(z) \frac{\partial \theta}{\partial z}\right|_{z=H}=\eta\left(\theta_{a}-\theta\right) .
\end{aligned}
$$

Здесь $\theta(x, y, z, t)$ - концентрация примеси; $u, v$ - составляющие скоростей движения частиц по горизонтали в пограничном слое атмосферы, $u_{b}, v_{b}, w_{b}$ - скорости ветра в горизонтальном и вертикальном направлениях; $\kappa(z)$ коэффициент турбулентности, $w_{\mathrm{g}}-$ скорость осаждения частиц, $\mu$ - коэффициент диффузии по горизонтали, $\sigma$ - коэффициент поглощения, $\beta$ - коэффициент взаимодействия с подстилающей поверхностью, $Q_{0}-$ надземный источник выброса вредных веществ в атмосферу; $\eta$ эмпирический параметр, $Q-$ мощность точечного источника; $\delta_{i, j}-$ функция Дирака, $m$ - масса частицы; $r$ - радиус частицы; $t$ - время; $g$ 


\begin{tabular}{l|lr|ll|ll} 
& ISRA (India) & $=\mathbf{1 . 3 4 4}$ & SIS (USA) & $=\mathbf{0 . 9 1 2}$ & ICV (Poland) & $=\mathbf{6 . 6 3 0}$ \\
Impact Factor: & ISI (Dubai, UAE) $=\mathbf{0 . 8 2 9}$ & PUHЦ (Russia) $=\mathbf{0 . 1 5 6}$ & PIF (India) & $=\mathbf{1 . 9 4 0}$ \\
& GIF (Australia) & $\mathbf{0 . 5 6 4}$ & ESJI (KZ) & $=4.102$ & IBI (India) & $=\mathbf{4 . 2 6 0}$ \\
& JIF & $=\mathbf{1 . 5 0 0}$ & SJIF (Morocco) & $=2.031$ & & \\
\hline
\end{tabular}

- ускорения свободного падения; $c_{f}$ коэффициент лобового сопротивления частиц; $k_{f}$ - коэффициент формы тела для силы сопротивления; $F_{n}$ - подъемная сила воздушного потока; $\rho_{n}-$ плотность частиц; $\rho_{\theta}-$ плотность воздуха; $\mu_{6}-$ вязкость воздуха.

\section{Метод решения}

Систему (16)-(23) интегрировать Задачу (1)(4) рассмотрим в области $D=(0<x<a, 0<y<b$, $0<z<H), \quad$ a источник предполагаем расположенным в приземном слое.

Величина $Q 0$ является функцией от $x, y, z, t$ и она вычисляется в ходе решения задачи, связанной с эрозией почвы, которая зависит от метеорологических условий и свойств подстилающей поверхности, размера и плотности пылевых частиц [13-14].

Для интегрирования поставленной задачи основные параметры математической модели процесса $u_{b}, v_{b}, w_{b}, k, \mu$ будем определять в виде степенных функций [15]

$$
\begin{gathered}
u_{b}(z, t)=\left|v_{1}\right|\left(\frac{z}{z_{1}}\right)^{n} \cos \alpha, \\
v_{b}(z, t)=\left|v_{1}\right|\left(\frac{z}{z_{1}}\right)^{n}, \\
w_{b}(z, t)=\left|v_{1}\right|\left(\frac{z}{z_{1}}\right)^{n} \sin \alpha, \\
k(z, t)=k_{0}(z, t)+\bar{k}(z, t), \\
\mu=|\vec{v}|\left(k_{x}+R \theta_{0}^{2}\right),
\end{gathered}
$$

где $\left|v_{1}\right|$ - модуль скорости ветра при $z=1$ м, $\alpha$ - параметр, указывающий направление ветра относительно горизонта.

Составлявшей ветра вычисляются с помощью формулы

$$
\mathrm{U}=\left(\mathrm{u}_{b}^{2}+v_{b}^{2}+w_{b}^{2}\right)^{1 / 2} .
$$

Надо отметить, что в работах многих авторов коэффициент взаимодействия с подстилающей поверхностью $\beta$ изменяется в пределах $0 \leq \beta \leq 1$. Если частица попадает в твердую стенку, то считается $\beta=0$, если ударяется о водную поверхность, то $\beta=1$. Обычно часть аэрозольных выбросов в зависимости от своей массы, метеорологических и климатических условий, характеристик подстилающей поверхности и географического расположения области распространения попадает на подстилающую поверхность. Затем часть из них снова попадает в атмосферу. Считают, что $\beta$ является постоянной величиной в рассматриваемой области. Это искажает прогноз распределения частиц на подстилающей поверхности. Чтобы избежать такой проблемы, при разработке математической модели процесса нужно учитывать $\beta=\beta(x, y, z)$.

Для учета поглощения аэрозольных частиц в растительном покрове коэффициент взаимодействия с подстилающей поверхностью $\beta$ необходимо вычислять с помощью формулы $[14,16]$ :

$$
\begin{gathered}
\beta(x, y, z, r)=\left\{\begin{array}{cc}
0, & \mathrm{z}>\mathrm{z}_{k}, \\
0,264 w(z)^{1,65} w_{g}^{0,66} s(z), & z<z_{h} .
\end{array}\right. \\
\text { Здесь } z_{h}-\text { высота слоя растительности, }
\end{gathered}
$$

$s(z)$ - удельная поверхность растительности.

Подъемная сила ветра определяется с помощью:

$$
F_{n}=k_{n} \pi r^{2} \rho_{B} \mathrm{U}^{2},
$$

а сила лобового сопротивления

$$
F_{\pi}=0,0078 \pi r^{2} \rho_{B} \mathrm{U}^{2},
$$

где $k_{n}-$ коэффициент подъемной силы воздуха который изменяется в пределах от 0,03 до 0,18 (для почвы и песка 0,09-0,18).

Мощность надземного источника вычисляется со помощью:

$$
Q_{0}=\frac{0.12 \theta_{n} g}{\rho_{n} P} Q_{0}^{\prime},
$$

а расход примесей

$$
Q_{0}{ }^{\prime}=\frac{c \rho_{B} u_{*}^{2}}{g}\left[1-\left(\frac{\mathrm{u}_{* \mathrm{kp}}}{\mathrm{u}_{*}}\right)^{2}\right],
$$

где

$$
c=0.25+\frac{w_{g}}{u_{*}} .
$$

Критическая скорость трения вычисляется по формуле

$$
\mathrm{u}_{* \mathrm{kp}}=\sqrt{0,0123\left(\frac{\rho_{B}}{\rho_{n}} d g+\frac{3 \cdot 10^{4} c^{2}}{\rho_{n} d}\right)} .
$$

При больших скоростях ветра существует простая зависимость скорости трения $\mathrm{u}_{*}$ от средней скорости ветра $\mathrm{u}_{2}$ : $\left(\mathrm{u}_{*}-\mathrm{u}_{* \mathrm{kp}}\right)=0.44\left(\mathrm{u}_{2}-\mathrm{u}_{2 \mathrm{kp}}\right)$.

Здесь $\mathrm{u}_{\text {*kp }}=45 c м / c ; \mathrm{u}_{2 \mathrm{kp}}=7 c$ м $/ c$. При пылевых бурях $\mathrm{u}_{*}$ меняется от 50 до $80 \mathrm{~cm} / \mathrm{c}$.

\section{Выводы}

Для мониторинга и прогнозирования экологических состояния промышленных регионов в отличие от рассмотренных выше работ была разработана математическая модель процесса переноса и диффузии вредных 
мелкодисперсных частиц, в которой учитываются существенные параметры объекта исследования: скорость перемещения частиц аэрозольных выбросов в атмосфере, которая зависит от массы и радиуса частиц; ускорение свободного падения частиц; коэффициент лобового сопротивления частиц при переносе их воздушной массой атмосферы; коэффициент формы тела для вычисления силы сопротивления; подъемная сила воздушного потока; $\rho_{n}-$ плотность частиц и воздуха; $\mu_{в}-$ вязкость воздуха.
Предложенную математическую модель можно использовать для достоверного прогноза состояния воздушного бассейна промышленных регионов, вследствие выброса вредных веществ из объектов производства и в следствия эрозии постилающей поверхности земли, а также для оптимального разрешения новых производственных объектов.

\section{References:}

1. Arushanov M.L., Teleumuratova B.S. (2012) Dinamika ekologicheskikh protsessov Yuzhnogo Priaral'ya/ Gamburg : Palmarium. 2012 . - 183 p.

2. Teleumuratova B.S. (2009) Vliyanie solepyleperenosa na osadkoobrazovaniya $\mathrm{V}$ Priaral'e/Aridnye ekosistemy -2009 . - tom 15. № 3(39). p. 28-35

3. Teleumuratova B. S. (2007) Vliyanie sul'fitnogo aerozolya na klimaticheskie kharakteristiki yuzhnogo Priaral'ya//Vestnik KKO AN RUz. - 2007 .- № 2. -p. 5-8

4. Teleumuratova B.S. (2004) Matematicheskoe modelirovanie perenosa aerozolya $\mathrm{v}$ nizhney sloyakh atmosfery/ Diss. kand. fiz.-mat. nauk . Tashkent, 2004. -138 p.

5. Pushilina Yu.N. (2011) Sovershenstvovanie metodov modelirovaniya i monitoringa zagryazneniya atmosfernogo vozdukha gornopromyshlennogo regiona: Dis. ... kand. tekhn. nauk. - Tula, 2011. - 156 p.

6. Radkevich A.V, Bieliaiev M.M., Stepanenko O.O., Yakovlev S.O., Shaptala O.I., Lysniak V.M. (2012) Calculation of air pollution in case of an accident based on the model convective and diffusion of the pollutant transfer // Springer Science \& Business Media. - 2012. 298 p.

7. Seregina O.V. (2011) Otsenka pylegazovykh vybrosov $\mathrm{v}$ atmosferu ugol'nymi shakhtami i sovershenstvovanie otraslevoy metodiki inventarizatsii istochnikov zagryazneniya: Dis. ... kand. tekhn. nauk. - Tula, 2011. - 147 p.

8. Meskhi B.Ch., Maslov E.I., Solov'ev A.N., Bulygin Yu.I., Koronchik D.A. (2011) Matematicheskoe i eksperimental'noe modelirovanie protsessov rasprostraneniya oksidov ugleroda i izbytkov teploty $\mathrm{v}$ gazovozdushnoy srede pomeshcheniya // Vestnik DGTU. - 2011. - T.11, № 6. - p. 862874.

9. Lisenko, S.A (2018) A Fast Algorithm for Retrieving Maps of Atmospheric Pollution by Fine Particulate Matter from Multispectral Satellite Images//Atmospheric and oceanic optics, jan 2018, Tom: 31, 60-71, DOI: 10.1134/S1024856018010104

10. Elperin, T., Fominykh, A., Katra, I., Krasovitov, B. (2017) Modeling of gas adsorption by aerosol plumes emitted from industrial sources// Process safety and environmental protection.-Tom: 111,pp.375387DOI: 10.1016/j.psep.2017.06.022

11. Vil'fand, R.M., Kirsanov, A.A., Revokatova, A.P. Rivin, G.S., Surkova, G.V. (2017) Forecasting the transport and transformation of atmospheric pollutants with the COSMO-ART model// Russian meteorology and hydrology. tom 42, s. 292-298, - may 2017, DOI: $10.3103 / \mathrm{S} 106837391705003 \mathrm{X}$

12. Elperin, T., Fominykh, A., Krasovitov, B. (2016) Effect of raindrop size distribution on scavenging of aerosol particles from Gaussian air pollution plumes and puffs in turbulent atmosphere// Process safety and environmental protection. Tom: 102. Str.: 303-315 . DOI: 10.1016/j.psep.2016.04.001

13. Ravshanov N. Narzullaeva N. (2017) Modelirovanie protsessa perenosa i diffuzii melkodispersnykh chastits $\mathrm{v}$ atmosfere $\mathrm{s}$ uchetom erozii pochvy// Problemy vychislitel'noy i prikladnoy matematiki. Tashkent, 2017. - №4(10). - p. 19-35.

14. Ravshanov N., Toshtemirova N.N. (2016) Protsessy perenosa i diffuzii aerozol'nykh chastits v atmosfere: metodologiya 
\begin{tabular}{l|lr|ll|ll} 
& ISRA (India) & $=\mathbf{1 . 3 4 4}$ & SIS (USA) & $=\mathbf{0 . 9 1 2}$ & ICV (Poland) & $=\mathbf{6 . 6 3 0}$ \\
Impact Factor: & ISI (Dubai, UAE) $=\mathbf{0 . 8 2 9}$ & PUHЦ (Russia) $=\mathbf{0 . 1 5 6}$ & PIF (India) & $=\mathbf{1 . 9 4 0}$ \\
& GIF (Australia) & $\mathbf{0 . 5 6 4}$ & ESJI (KZ) & $=4.102$ & IBI (India) & $=\mathbf{4 . 2 6 0}$ \\
& JIF & $=\mathbf{1 . 5 0 0}$ & SJIF (Morocco) & $=2.031$ & & \\
\hline
\end{tabular}

komp'yuternogo modelirovaniya. Saarbrücken (Deutschland): LAP Lambert Academic Publishing, 2016. $-136 \mathrm{p}$.

15. Sharipov D.K., Toshtemirova N., Narzullayeva N. (2016) Numerical modeling of the spread of harmful substances in the atmosphere taking into account terrain // Problems of computational and applied mathematics. Tashkent, 2016. - №1(3). - p. 60-71.
16. Ravshanov, N. (2015) A Mathematical Model for the Study and Forecast of the Concentration of Harmful Substances in the Atmosphere / N. Ravshanov, M. Shertaev, N. Toshtemirova // American Journal of Modeling and Optimization. - 2015. - Vol. 3, No. 2. - p. 3539. 